\section{UNA BOZZA PER UN' OPERA TEATRALE DI LEONORA CARRINGTON E REMEDIOS VARO}

A DRAFT FOR A PLAY BY LEONORA CARRINGTON AND REMEDIOS VARO

\section{RIASSUNTO:}

Si propone in questo saggio una breve analisi In this paper is proposed a brief analysis of a di una bozza per opera teatrale scritta da draft for theatrical works written by two artists, due artiste, Leonora Carrington e Remedios Leonora Carrington and Remedios Varo, who Varo, che si sono distinte nel panorama have distinguished themselves in the cultural culturale delle avanguardie del novecento climate of the avant-garde from the Twentieth avvicinandosi al surrealismo per indicarne Century, approaching to surrealism to tematiche, ma anche le differenze rispetto al thematic reports, but also the movimento, svolgendo ognuna un percorso with this movement, each carrying a paralle

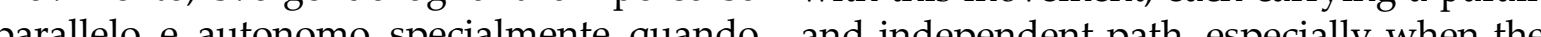
parallelo e autonomo specialmente quando and independent path, especially when they
migrano dal contesto europeo in Messico.

PAROLE CHIAVE:

Surrealismo, alchimia, ironia, conoscenza.

\section{KEY WORD:}

Surrealism, irony, draft, knowledge.
Nell'ambito degli studi relativi ad alcune artiste - scrittrici del novecento le storie di vita e l'iter creativo di Leonora Carrington e Remedios Varo appaiono come esperienze singolari di due 'donne in viaggio' che hanno gravitato nell'entourage dell'avanguardia surrealista tra gli anni Trenta e Quaranta, anche se non hanno aderito al movimento fondato da André Breton nel 1924, rinunciando ad ogni classificazione per testimoniarsi in presenza come figure indipendent e originali per la loro sperimentazione nella produzione pittorica e letteraria recentemente riscoperta. Quando giungono a Parigi nel 1937 presentano già una loro autonomia di linguaggio nei confronti delle presenze maschili quali esponenti autorevoli del nucleo surrealista. Leonora di origine inglese, Remedios di terra spagnola, preferiscono essere valutate per la propria individualità, come artiste e donne, ma primariamente come persone, al di là di ogni differenza di genere. ${ }^{1}$ Specialmente quando 'migrano' dall'Europa in Messico all'indoman dello scoppio del secondo conflitto mondiale tra il 1941 e il 1942 condividono esperienze dettate da un'autentica amicizia e manifestano nel loro lavoro creativo, come paesaggio dell'immaginario, gli stati interiori più profondi che si intrecciano con la dimensione quotidiana del vivere.

\section{CARTE DI IDENTITÀ APERTE}

Nata nel 1917 a Clayton Green, vicino a Chorley, nominato Black Country, Leonora Carrington appare fin dall'inizio insofferente nei confronti delle rigide convenzioni di una famiglia vittoriana benestante ancorata al perbenismo sociale e ad una rigorosa educazione conservatrice che non ammetteva la sua propensione artistica, in contrasto, quindi, con il suo spirito, la sua indole, il suo carattere ribelle e irriverente. ${ }^{2}$ L'ammissione all'Ozenfant Academy a Londra nel 1935, il fervido ambiente parigino, l'incontro con l'artista sciamano Max Ernst, ${ }^{3}$ in occasione di una sua mostra londinese, la prigionia di quest'ultimo in seguito allo

L'idea che le donne non fossero creative ma capaci di dedicarsi alla decorazione, all'imitazione, alle arti minori era ormai radicata nel mondo ottocentesco. Come sottolinea J. J. Spector :"The Surrealists only partially break out of this mold. [.... Believing in a universal equality at the level of dream and the unconscious (a level irrelevant to Marxist class conflict), the Surrealists developed a vision of humanity beyond divisions of gender, class or race, albeit as theoretical and poetic as the Romantic Hugo's"' (Spector, 1997:184)

2 Per Leonora il surrealismo incarna quindi "uno stato d'animo, una condizione dello spirito, come aspetto fantastico della realtà [...] che include l'esteriore e l'interiore" (Carrington, 2004, Sileo, 2007: 209) per esplorare gli spazi sotterranei della nostra psiche, per indagare nel mistero dell'esistenza.

3 Leonora instaura una relazione con Max, l'artista - sciamano, l'amante e il 'padre' che avrebbe voluto senza porsi problemi per la differenza di età. Quest'ultimo sarà obbligato nel 1939 a presentarsi al campo di concentramento per stranieri nel carcere di Largentiere in Ardeche, come tedesco in campo di Les Milles, perde nuovamente la libertà. 
scoppio del secondo conflitto mondiale che le comporterà di cadere in uno stato di prostrazione prossimo alla psicosi, segneranno la sua giovinezza, tanto da ricercare una via di fuga imbarcandosi per il Messico, sposando per convenienza il poeta e scrittore messicano Renato Leduc, ${ }^{4}$ una nuova meta oltreoceano che le permetterà di continuare la sua ricerca creativa.

Maria de los Remedios Alicia Rodriga Varo y Uranga nasce ad Anglés, un piccolo paese in provincia di Gerona, a nord di Barcellona, quasi al termine del primo decennio del novecento. ${ }^{5}$ Viaggiando per esigenze di lavoro del padre, conoscerà usi e costumi, culture diverse, che lasceranno una traccia nel suo itinerario creativo in verità mai osteggiato, anche se vigilanza, oppressione, necessità di fuga, sono le stesse tensioni e gli stessi desideri che prova Remedios specialmente quando viene iscritta ad una scuola religiosa dopo il trasferimento a Madrid nel 1917 Gli studi presso 1'Accademia San Fernando, la frequentazione degli ambient e dei gruppi d'avanguardia, come i Logicofobisti, amanti dell'irrazionalità, la conoscenza delle prime esperienze cinematografiche di Luis Buñuel e degli Amics de l'art nous (ADLAN) alla metà degli anni Trenta a Barcellona, dove incontra Benjamin Péret ${ }^{6}$ il poeta surrealista dell'amor sublime, fino al trasferimento con lui in Messico, come esilio per lei ${ }^{7}$ volontario, le permettono di attingere ad un repertorio di portati e di esperienze rilevanti per la sua vita intima e artistica La sua nuova abitazione nel calle Gabino Barreda diventa subito un luogo di

4 Leonora cade nello smarrimento totale e riuscirà a risolvere solo dopo l'internamento a Santander come racconta in Down Below, scritto a posteriori quasi per esorcizzare il suo stato di psicosi, la su discesa agli inferi necessaria per riassumere consapevolezza e confermare a se stessa la 'guarigione'. Uscita dall'istituto, per evitare di sottoporsi ad altre cure trasferendosi in South Africa secondo le decisioni paterne, prima di imbarcarsi a Lisbona riesce a raggiungere l'ambasciata messicana e chiedendo aiuto a Renato Leduc, poeta e scrittore di origine messicana conosciuto in precedenza, si sposerà con lui e partirà per il Messico (Aberth, 2010: 51)

5 Il nome Remedios, scelto dalla madre in onore della Virgen de los Remedios, come 'rimedio' alla morte di una precedente figlia, sicuramente determina un senso di frustrazione nella personalità dell'artista che si sentirà sempre una 'sostituta', mentre Rodriga è il nome imposto dalla tradizione familiare.

6 Il 'poeta dell'amor sublime', tra gli esponenti principali del movimento surrealista, diventa l'emblema della lotta per la liberazione materiale dell'umanità congiunta alla liberazione spirituale. Si contraddistingue per l'impegno politico di impronta di sinistra fin dai tempi dell'Ottobre rosso migrando in Brasile dove rimane dal 1929 al russa, anima l'opposizione e si schiera con Trotsky Rientrato in Francia, si confronta con la contraddittoria attività delle correnti trotskiste, mentre in Spagna a Barcellona nel 1936 si schiera con il fronte popolare repubblicano e milita per la ca Spagna, a Barcellona nel 1936 si schiera con il fronte popolare repubblicano e milita per la causa

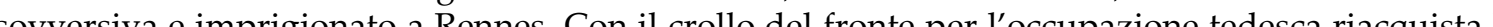
libertà e 1941 si ifugia a Marsigla, nella Francia 'libera' per poi migrate in Messico.

7 Non per Benjamin Péret, come menzionato nella nota precedente. Remedios approda nel 1941 a Veracruz dopo un arduo viaggio sul Serpa Pinto lungo la rotta che attraversa l'oceano da Casablanca, per stabilirsi a Città del Messico e capisce subito di essere giunta in un paese che le consente di poter zioni che si è lasciata alle spalle allontanandosi dalla terra di origine. confronto e di sperimentazione specialmente con Leonora, che aveva conosciuto due anni prima nella capitale francese in casa di Breton ${ }^{8}$ e con cui consolida un legame alimentato da un' affinità ${ }^{9}$ dovuta all'intensità di energie che ambedue emanano e di cui sono custodi, come scrive di loro Octavio Paz:

There are in Mexico two admirable artists, to bewitched witches: they have never heard the voices of praise or disapproval from schools and parties [...]. Untouched by social mores, by aesthetics and prices, Leonora Carrington and Remedios Varo move through our city with an air of supreme and ineffable distraction. Where are they going? Where their imagination and passion call them (Paz, 1990:296).

Messico è magia, archetipo, oggetto totemico, natura naturans. La diversità $\mathrm{d}$ un paese rispetto all'ambiente europeo che interpretano inizialmente come luogo per l'azzeramento di schemi preordinati, sperimentando nuove tecniche, Leonora dedicandosi a una nuova 'cosmologia pittorica' (Feman Orenstein, [1975], 1977: 66 - 77, cit. Agnati, 2003: 36) ${ }^{10}$, Remedios applicandosi nell'ambito di una 'cosmogonia figurativa eliocentrica'11 per cui l'icona umana è astro motore, protesa a cogliere e ad esprimere il prodigio dell'universo. Il deragliamento dai sistemi logici, la casualità, l'accostamento fortuito sono concepiti dalle due amiche in modalità ironica e divertita nei loro giochi sul cadavere squisito di impronta surrealista, mentre trascorrono ore a narrare o leggere le proprie storie una di seguito all'altra senza relazioni logiche, 'provocandosi' a vicenda effetti di spaesamento, o negli accostamenti di ingredienti e oggetti in modalità inconsuete, simili alle ricette magiche, inventate specialmente da

8 Si stabilisce nelle vicinanze lungo Rosa Moreno in un appartamento di una costruzione abbandonata dell'ambasciata russa.

9 Leonora approda a New York ma vivrà dal 1942 in Messico come Remedios, anche se, quest'ultima, nel 1947, in seguito alla separazione da Péret che ritornerà nella capitale francese, parte con il pilota Jean Nicolle per il Venezuela dove rimane per due anni partecipando anche a una spedizion scientifica francese di entomologia sul fiume Orinoco. E' tramite Remedios, inoltre, che Leonora conosce Emerico Weisz, soprannominato 'Chiki', un fotogiornalista ebreo ungherese amico di Breton, anche lui giunto in Messico con altri rifugiati di guerra da Casablanca, che diventerà più tardi il suo compagno fino alla scomparsa. "Todos $[. .$.$] pasaron en México bastante tiempo durante$ la guerra y después, y todos [...] habian estados relacionados con el movimento surrealista en Europa y permanecian leales a su espiritu. Juegos surrealistas, bromas pesadas, fiestas de complicados disfraces, el contar cuentos estridentes hasta bien entrada la noche" (Kaplan, 1998:89).

10 Remedios lavora come illustratrice tecnica per la Divisione di studi sulla malaria del Ministero della Salute pubblica in Maracay per la ricerca di batteri patogeni e per il laboratorio farmaceutico Bayer in Messico

11 Diego Sileo propone due livelli di trascendenza nell'itinerario di Remedios Varo relativa alle reti occulte di legami che l'occhio non riesce a vedere e all'apertura di un varco per scappare dalla tram come esperienza radicale e definitiva (Sileo, 2007: 97). 
Remedios $^{12}$ tanto da provocare sogni erotici e visioni, similmente a Salvador Dalì che conia il metodo della paranoia critica.

\subsection{La bozza: libertà e divertissement}

Tra gli scritti di Remedios Varo che non hanno mai raggiunto una forma organica, conservati nell'archivio del suo ultimo compagno, Walter Gruen, recentemente scomparso, come testimonianze diaristiche o appunti stesi in quaderni diversi in modo similare alla scrittura automatica, accompagnati da un torrente di immagini, compaiono indicazioni sommarie o spunti relativi a visioni ricette per curare l'insonnia e per indurre sogni, ma anche una bozza per opera di teatro scritta a due mani con Leonora probabilmente dopo la metà degli ann Quaranta, quando ambedue vivono in Messico, anche se risulta difficile stabilire la data di elaborazione. ${ }^{13}$

Ella [Varo] y Carrington ideaban toda clase de juegos, experimentos, historias y obras de teatro de los que rebosan los cuadernos de Varo. - scrive Janet Kaplan-Con una abierta afición a los cuentos de hadas, colaboraron en una farsa escatológica muy divertida [...] a la que le dieron la forma de una complicada obra de teatro en tres actos, con directrices para la puesta en escena y finales alternativos, en la que había papeles para muchos de sus amigos (Kaplan, 1998: 96).

Presenta tre scritture autografe a penna, corrispondenti a Remedios, a Leonora, che intervengono alternativamente nella stesura creata probabilmente in simultaneità, e a Kati Horna, una fotografa ungherese loro amica, che inserisce solo una nota preliminare e due didascalie sicuramente aggiunte dopo l'elaborazione del testo e la lettura finale. ${ }^{14}$ Si distingue come creazione di puro divertissement, come dichiara Kati nella nota menzionata: "Cualquier parecido del publico con algun personaje, es pura coincidencia. Esta obra esta hecha solamente para diversion de los actores. El publico es puro accidente". Non è dato sapere se sia stata rappresentata o solo letta nella cerchia delle amicizie delle due

12 "Un viejo directorio telefónico, un ramo de laurel, una chinche, un peine, un bote de pintura verde, un zapato de mujer de terciopelo violeta bordado de perlas y una moneda falsa de cinco peso [...], ciertos incidentes se han producido [...]: mi mejor camisa se ha quemado, un gran depósito de sal se ha acumulado debajo de mi cama, y al día siguiente tuvo lugar el inicio de la sorprendente transformación de mi inspiración pictórica" (Varo, Carta 6, Castells, [1994], 1997:38).

13 “Todos estos eventos (cfr. nota 9) nos llevan a formular la hipótesis de que la obra teatral pudo

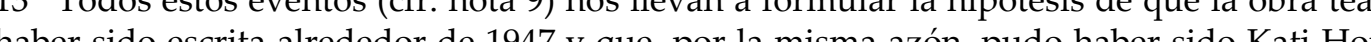
haber sido escrita alrededor de 1947 y que, por la misma azon, pudo haber sido Kati Horna quien
inicialmente conservara este manuscrito" (Mendoza Bolio, 2010:104).

14 Una delle due note che afferma come Kati Horna abbia partecipato 'en el juego', appare nella scena seconda del primo atto, in cui aggiunge l'aggettivo 'loca' ("Con una risa ligera y blanca de virgen loca") e nell'atto II scena II ("El orazón es verdadero"), (Mendoza Bolio, 2010: 105). artiste, anche se Edith Mendoza Bolio ${ }^{15}$ presume che fosse di carattere privato e sottolinea la stretta relazione con le tecniche surrealiste. Infatti ambedue le artiste - scrittrici non manifestano intenzioni letterarie e prediligono la casualità come impronta o paradigma iniziale che rinvia all'automatismo psichico, alla scrittura automatica; non prevedono inoltre il rispetto della punteggiatura, sulla linea delle suggestioni bretoniane, ${ }^{16}$ anche se, in verità, il testo senza titolo assume forma compiuta, distinto in tre atti, di cui l'ultimo scritto sul recto e sul verso de foglio e elaborato anche come seconda versione incompiuta da Remedios con alcuni schizzi, abbozzi e disegni. Il carattere ludico e ironico che trapela dal testo come dall'opera pittorica di ambedue, si collega alle loro intenzioni di lavorare in libertà come gioco gratuito, come 'intermezzo nella vita quotidiana'(Huizinga, 1957: 16), per cui non si attengono scrupolosamente alle regole linguistiche, come emerge nelle parti scritte in lingua spagnola da Leonora, e preferiscono il gioco di simulazione, basandosi sullo scambio dei ruoli, per cui i personaggi maschil interpretano figure femminili e viceversa, ognuno temporaneamente sulla scena cambia identità, diventa altro da sé nell'ottica del travestimento.

\subsubsection{I personaggi}

En la lista de los personajes - dramatis personae - las autoras incluyen los nombres dequienes participarían en la obra:Chiki Weisz, esposo de Carrington, representaría a la reina Nesfatalina [...]- ‘Dama Blanca del Pico de Oripí José Horna, esposo de Kati Horna, aparecería en scena como la princesa Pelomiel, [...] "dulce paloma palida y bellisima hija de Nesfatalina; Remedios Varo en el papel de Scatijeras, el cientifico de la corte, y Carrington en el del fiel asistente de la Reina, Perico Verde [...] "alma de un difunto diplomatico". En cuanto a Jean Nicolle ${ }^{17}$ sería el único en encarnar a un personaje de su mismo género y oficio, don Jon von Aguilota, que al igual que él, era un piloto aviador (Mendoza Bolio, 2010: 108).

Il mutamento di identità tramite il travestimento tipico nella finzione scenica fin dall'antichità, rinvia, in tal caso, al metamorfismo, quale componente

15 Nelle Conclusiones la studiosa purtroppo prematuramente scomparsa nel 2012, che ha il merito di aver esaminato e riscoperto $i$ testi scritti di Remedios e di aver provveduto all'edizione critica scrive: " La obra de teatro en colaboración con Carrington es un manuscrito que nos ha permitido presentar su edición en dos versiones: la edición genética que posibilita rescatar el proceso de escritura y conservar el escrito lo más cercano posible a su versión original, con el fin de difundirla a un público más amplio; y la edición crítica que presenta la pieza teatral depurada. [...]. Varo y Carrington escribieron como si jugaran y con [El Santo Cuerpo Grasoso] dieron origen a un mundo posible, surreal y lleno de fantasía" (Mendoza Bolio, 2010:136).

16 André Breton e Philippe Soupault nel primo manifesto del surrealismo indicano, avendo iniziato a Acarabochiare, il “lodevole disprezzo per le conseguenze letterarie” e come "la punendo iniziato (1) opponga alla assoluta continuita de ${ }^{2}$ lusso verbale, anche se sembra cosi indispensabile come la
distribuzione dei nodi in una corda vibrante" (Breton, 2001: 42, 49). 17 Jean Nicolle, come menzionato nella nota 9 è un compagno di Remedios negli anni in cui si reca in Venezuela. 
singolare nell'itinerario creativo di Leonora e Remedios e caratteristica propria dell'universo artistico surrealista, ma anche all'ambiguità dell'androgino, in cui si incontrano gli opposti, maschile e femminile, figura emblematica del processo alchemico che nel manoscritto per opera teatrale diventa il tema principale come nelle loro opere pittoriche in relazione al topos del viaggio alla ricerca della verità come acquisizione della conoscenza, veggenza, saggezza del vivere, attraverso la trasmutazione e il superamento delle prove nella forma anche di esperimenti alchemici e formule magiche oracolari e misteriose che attivano un itinerario catartico e rivelatorio.

\subsubsection{L'incipit}

L'azione scenica si apre con l'uomo di scienza, Scatijeras, addetto culturale presso la corte della regina Nesfatalina.

Entra Scatijeras con aspecto de conspirador, juega nerviosamente con su larga barba roja. Salen de aquella cascada de pelos colorados algunas moscas grandes; empieza a mirar atentamente un frasquito que tiene en la mano (Carrington, Varo, ms in Mendoza Bolio, 2010: 234).

Compare proferendo queste parole: "Elíxir divino! Aceite celestial! Fruta mágica de mi inmenso saber! Oh, humanidad bestial, tiembla delante [de] este Santo Cuerpo Grasoso!" (Carrington, Varo, ms in Mendoza Bolio, 2010: 234).

Scatijeras è il vaticinatore, il mago, il saggio, l'uomo della sapienza alchemica, intento a preparare pozioni per giungere a scoprire e conservare un elisir, il "Santo Cuerpo Grasoso", citato nella battuta e adottato da Mendoza Bolio per titolare la bozza. E'esaltato da Scatijeras come il Sacro Graal, pietra filosofale e cibo sapienziale, ${ }^{18}$ olio sacrale, sostanza prima della scoperta e ultima della esplorazione che possiede la proprietà di separare l'anima dal corpo e di renderli indipendenti e visibili. ${ }^{19}$ La sua barba rossa è simbolo di saggezza, di raggiungimento della rubedo e viene rappresentato sulla scena proprio da Remedios il cui itinerario creativo è considerato un exemplum di un percorso interiore orientato verso la conoscenza di sé e del mondo come assunzione di consapevolezza, per migliorarsi, rigenerarsi, purificarsi fino all'annullamento e morte dell'io per rinascere.

\subsubsection{Sinossi e temi}

18 La regina Nesfatalina dice nella seconda scena dell'atto secondo che "Scatijeras ha descubierto una línea ancestorial directa entre las solitarias presidenciales y los originales cocodrilos sagrados de Toth, excellent familias Todos" (Carrington, Varo, ms in Mendoza Bolio, 2010: 243).

19 Nella mescolanza alchemica dalla nigredo all'albedo, dalla citrinitas alla rubedo, si vanifica la materia bruta per giungere alla quintessenza, mentre l'evaporazione è l'ultima fase o stadio del dell'opera dedicata alla Sposa. Il corpo oleoso, "grasoso", è come l'elemento "gaseoso", gassoso.
Riguardo alla sinossi dell'opera, in sintesi il saggio Scatijeras, che ha scoperto un elisir, desidera presentarlo alla regina Nesfatalina che bandisce un concorso per scegliere un' "alma hermosa" per la figlia Pelomiel, ma al contempo si presenta Jon von Aguilota, un pilota atterrato per un incidente con il suo aereo, che riconosce il saggio incontrato anzitempo, quando quest'ultimo si trovava alla corte di Rey Prisma $1^{\circ}$, da cui era stato espulso e perseguitato per un errore insignificante..$^{20} \mathrm{Il}$ pilota prova una passione per la principessa Pelomiel dopo averla vista, per cui ingannando la regina ruba l'elisir uccidendo il saggio Scatijeras per scappare con lei. Il Perico rivela ciò che è avvenuto e inizia la ricerca di Jon von Aguilota in fuga che viene imprigionato e che, in cambio della libertà, cede la sua anima e la sua fisionomia al Chango Nigro, anima bruta ${ }^{21}$ di Scatijeras che, nel frattempo ritornato in vita, ne assume le sembianze per presentarsi a Pelomiel durante i preparativi della gara, risultandone il vincitore. ${ }^{22}$ I temi che emergono e si alternano nel testo quali il viaggio come processo alchemico alla ricerca della verità che si intreccia con l'amore e l'erotismo, manifestazione dell'onnipotenza del desiderio, gli elementi che ne conseguono, quali la trasfigurazione e la trasmutazione alchemica attraverso il liquido magico e sapienziale, il metamorfismo, la chiaroveggenza l'occulto, l'humor negro o l'ironia nell'attesa della trasformazione, il mito de veggente e della dea luna diana - diviana e dea madre che si incarna nella figura femminile, il mistero tra esoterismo e occultismo, sono ricorrenti anche nell'opera pittorica delle due artiste.

\subsubsection{Le funzioni}

Tale bozza teatrale è stata considerata un exemplum di narrazione fantastica indagandone gli aspetti della trama secondo le funzioni tipiche della fiaba di Vladimir J. Propp così da individuare le fasi di un percorso di formazione compiuto dall'eroina cercatrice, $^{23}$ dallo stato di felicità della situazione iniziale proposta attraverso l'invenzione di Scatijeras, alla proibizione, alla provocazione del danno quando, in

20 L'errore consisteva nel fatto che aveva scambiato elisir, cosicché "debía dar al rey tres gotas del elíxir de "Larga vista" y le di tres gotas del elíxir de "Exuberante vegetación" (Carrington, Varo, ms in Mendoza Bolio, 2010: 238).

21 All'eroe cercatore, dopo essere stato ucciso dall'antagonista, il pilota Jon von Aguilota, si presenta el Changuito o el Chango nigro: "Scatijeras, soy tu alma. Vengo de [los] bosques primordiales para ocupar tu ser", (Atto II, Scena V) (Carrington, Varo, ms in Mendoza Bolio, 2010: 247). E' la sua anima in forma animale, simbolo del fuoco o del tuono nella cultura yoruba, anche nei riti americani, che compare poi di fronte a von Aguilota e che ride "agudamente", quando gli altri lo vedono morto

22 Nella seconda versione dell'atto terzo scritta sul verso del manoscritto da Remedios la regina desidera porre termine alla gara durata venticinque anni a cui partecipa Scatijeras tornato in vita in incognito.

23 Edith Mendoza Bolio si è interessata a tale analisi, mentre Dina Comisarenco Mirkin ha esaminato il percorso pittorico di Remedios Varo alla luce degli studi di Vladimir J. Propp e del mitologo Joseph Campbell. 
seguito alla caduta di un aereo sulla montagna l'antagonista, il pilota Von Aguilota, giunge a turbare la pace, traendo in inganno le vittime, la Reina Nesfatalina e Scatijeras stesso, che si lasciano convincere con i suoi falsi elogi ${ }^{24}$ fino a rubare e uccidere ${ }^{25}$ per ottenere ciò che desidera. Ne consegue la mediazione dell'eroe, Scatijeras, che riesce indirettamente a recuperare l'oggetto del desiderio riparando il danno, a riconquistare un corpo da combinare alla propria anima con un camuffamento ${ }^{26}$ per riacquistare la vita, giungere alla vittoria, allo stato di grazia mentre la partenza di Von Aguilota vittima e donatore, è accompagnata da un canto degli "zopilotes" simile a una nenia che riassume in modo grottesco gli eventi negativi nei toni comici tipici della commedia ${ }^{27}$

\subsubsection{L'ironia}

L'eroe cercatore, Scatijeras, partecipa ad un processo di trasformazione, di morte e rinascita, di reincarnazione, di mutamento, annullamento e resurrectio, fasi proprie del processo alchemico che avviene attraverso "el frotar las nalgas con l'aceite", "el santo Cuerpo Grasoso" che possiede un potere miracoloso ${ }^{28}$ per ottenere la separazione dell' anima dal corpo. "Strofinare le natiche" è un gesto scaramantico e propiziatorio e al tempo stesso ironico e irriverente, che suscita ilarità, comicità scherzosa nel grottesco,

24 "Reina blanca, pálida y divina luna de todos [los] cielos, incomparabile Nesfatalina!", dice alla Scatijeras! Siento una palpitante amistad por til'" (Carrington, Varo ms in Mendoza Bolio, 2010: 243, 245).

25 Provoca un danno perché uccide Scatijeras per rubare l'elisir: "Scatjieras se arrima por la terraza y en el acto el piloto le da un terrible trancazo en la cabeza con una botella de tequila vacía. [... Después, con un grito de triunfo, saca la sagrada botella del Santo Cuerpo Grasoso" (Carrington (24)

26 “Todos me creen muerto - dice Scatijeras, che, ritornato in vita grazie ad alcuni esperimenti occulti, gioca sul camuffamento, - Verdad es que sin la ayuda del venerable ermitaño que robó mi cuerpo de sepulcro para hacer sobre el ciertos experimentos coronados por el exito, ahora continuaria muerto. Con este disfraz nadie me conocerá" (Atto terzo, Scena I); "Ya estoy libre de este viejo cáscara"; Dice el Chango (brincando y tocando cascabeles) Ya no vive! Ole! Ya no vive! Ole! Ole!", (Atto II scena V e VI) (Carrington, Varo ms in Mendoza Bolio, 2010: 248 - 250).

27 "Por la culpa culpita de un guapo piloto,/ el gran Scatijeras rindió su alma./ Tra la, tra la, tra la,/ por su culpa culpita,/ Nesfatalina/ pierde su reino/ sin el Santo Cuerpo Gordo//Tra la, tra la, Pelomiel, palomita/ su amor culpable/ por el aviador Aguilota/ es la perdición de todos, Tra la, tra la." (Carrington, Varo ms in Mendoza Bolio, 2010: 246).

28 “Entra el Perico verde humildemente. Perico: Oh, Scatijeras, emperador de la divina inteligencia, tira este aceite demoniacal!. Scatijeras: Callado asqueroso pájaro, qué sabes tu de mis invenciones divinas, cerebro de periquito en un cuerpo color de lechuga? Perico: Gran Scatijeras, yo sé los poderes de esta pequeña botella: basta frotar las nalgas con el aceite para que el alma del humano salga visiblemente del cuerpo y los dos sigan viviendo cada uno por su lado" (Carrington, Varo ms in Mendoza Bolio, 2010: 234). Al termine del "frotar" si origina una farfalla che può esprimere la leggerezza del processo finale di trasmutazione e la levità dell'anima. Segno che il processo alchemico si è compiuto poiché el Chango nigro alla ricerca di un corpo, 'mercanteggia'29 per assumere gli stessi tratti fisiognomici dell'assassino. Esprime, altresì, la potenza energetica del desiderio che si combina con la componente erotica ${ }^{30} \mathrm{Il}$ gusto per il grottesco si manifesta di nuovo quando Scatijeras si ripresenterà vivo per ottenere la principessa nelle vesti $\mathrm{d}$ von Aguilota e i partecipanti alla gara indetta dalla regina per scegliere l'anima più bella dovranno recuperare le proprie in un enorme mucchio da cui vengono propriamente lanciate nella confusione generale. ${ }^{31}$

1.1.6. Il processo alchemico e la veggenza

L'anima, allora, diventa protagonista nella trasfigurazione e trasmutazione mentre traspare la sottile irrisione nei confronti delle conoscenze scientifiche i cui procediment e rimedi esprimono solo l'impotenza di fronte alle arti magiche, alla sapienza del veggente. Quando la Regina chiama a corte il Doctor Pon Fruta, “Cirujano del Illustre Facultad de Medicina de la Ciudad de Cacatorres" per "aplicar artos instrumentos medicinales", questi si arrende di fronte ai poteri occulti e divinatori. Addirittura per sapere ciò che è avvenuto dal Perico presente alla 'tragedia', tenta l'ipnosi, una tecnica che trova riferimenti con la psicanalisi, il mesmerismo, itinerari percorsi dai surrealisti alla scoperta dell'ignoto e dell'inconscio. Sembra pronunciare una formula magica come un veggente ma senza esiti ("Hipna Hipnosis! Ora Pro Dosis"), mentre il Chango nigro conosce le vie dell'oltre, alimenta il processo di resurrectio e si avvicenda per "el cambio" alla prigione, per incontrare von Aguilota, con due trecce lunghe, un volto di color amarillo, e una barba nera, il colore della nigredo alchemica e del profondo. ${ }^{32} \mathrm{Un}^{\prime}$ altra figura oracolare e misteriosa è la donna luna cui allude nella bozza dell'opera tragicomica e escatologica il nome della regina, Nesfatalina, anche se la sua potenza energetica si configura come potenzialità del desiderio erotico, diventando

29 Il ritorno in incognito dell'eroe si presenta secondo Edith Mendoza Bolio quando "Arranca su barba negra de puntitas y la da al piloto tomando los bigotes colibriados y se los pone...", svolgendo un difficile compito, la competizione delle anime, per giungere al compimento, attraverso 1 rasfigurazione umoristica, "Ah! Ahora, piloto seductor, primero sus bigotes..; Ahora su alma!" (Carrington, Varo, ms in Mendoza Bolio, 2010: 255 - 256) per terminare con il castigo dell'antagonista e con le nozze.

30 "Que le pinchan las nalgas cómo lo demas! Luego veremos". "Tra la la. Tra la la,/ Una nalga, nada más!/Tra la la! Tra la la!/ Una nalga nada más!" (Carrington, Varo, ms in Mendoza Bolio, 2010: $242,293)$ Dice Nesafatalina: "Piloto von Aguilota, ser exquisito, le espero dentro de una hora en mi apartamentos privados" ((Carrington, Varo, $\mathrm{ms}$ in Mendoza Bolio, 2010: 244)

31 “Pasen! Pasen! Se ñores! No tengan miedo! Entreguen sus almas sin cuidado! - dice il Perrico - [...] mentre, come indicato nei suggerimenti di regia - los zopilotes y el cirujano buscan desesperadamente en el montón de almas - e lui - impieza a lanzar las almas al furibundo multitud que los regresan en el montón de almo - lermin (Carrington, Varo ms in Mendoza Bolio, 2010: 258).

32 Scatijeras "sacando el changuito negro de su bolsa, Está ya disminuído al tamaño de una zanahoria - Qué feo eres! Pero no le hace, ja! ja! ja! Vamos a hacer un pequeño cambio! Ja! Ja! Ja. El alma del Gran Scatijeras será digna del cuerpo. Maldito changuito! Je,je,je!", (Carrington, Varo ms in Mendoza Bolio 2010: 254). 
interprete di alcune scene che assumono un risvolto comico per la incapacità di trattenersi, affidandosi agli istinti. ${ }^{33}$ La pulsione erotica, che, in verità, esprime la potenza onnipresente, eversiva e inesauribile del desiderio proprio del surrealismo, $\mathrm{s}$ combina con l'ironia dello sguardo e il percorso alchemico, come aspetti di un'opera teatrale che rispecchia fondamentalmente una componente autobiografica in completa autonomia rispetto ai portati del surrealismo orientato verso l'alchimia e l'esoterismo come occultamento profondo attraverso le parole e le convinzioni di André Breton che invitava a riflettere sull" Alchimia del verbo' in occasione della pubblicazione del secondo manifesto nel 1929.

1.1.7. Animali totemici e ambienti primordiali

Compaiono ad arricchire la bozza teatrale gli animali come emblemi totemici, presenze inquietanti o protettrici in cui si riflette l'universo interiore dell'immaginario di ambedue, dalla scimmia, el Chango nigro, simbolo dell'istinto naturale, che coincide però in senso esoterico con l'anima 'bruta', nera, oscura, ai pipistrelli, uccelli sinistri ma emblemi di rinascita interiore nella notte alchemica, della nigredo, ai conigli, intuitivi messaggeri lunari; dalla gallina, che rinvia al calore moderato e costante del fuoco alchemico per passare nella mescolanza dei metalli dallo stato del piombo all'oro, alla farfalla simbolo di trasformazione e cambiamento, al gatto, messaggero divino e sacro nell'antico Egitto, simbolo di oscurità, morte, follia, ma anche di veggenza come la civetta, ${ }^{34}$ il gufo, che esprimono nella loro ambivalenza i medesimi significati simbolici prediletti specialmente da Remedios proprio per la potenza dello sguardo, la capacità di vedere oltre. Campeggiano negli spazi dell'immaginario delle opere sia pittoriche che letterarie, quindi anche negli ambienti scenografici di [El cuerpo grasoso], in cui nel primo atto il centro di un cratere, (dell'Oropipì) nel paesaggio tellurico diventa un autentico luogo magico, alchemico e primordiale, alle origini e radici della terra, come il pozzo di luce ne Il cornetto acustico di Leonora o la caverna misteriosa e oscura ne Il vuelo

33 La donna luna possiede e si alimenta di energie cosmiche per assumere poteri superiori, figura analogica e archetipica della dea Diana - Diviana e della Crande Madre, immagine totemigara percorso pittorico di ambedue le artiste. Nell'atto II scena II, senza curarsi del sistema di corte, Nesfatalina agisce al di là delle convenzioni, poiché "con un agilidad sorprendente [... brinca encima de Aguilota", che la invita a frenarsi: "Momentito! Momentito! Majestad [. . ] No estamo encima de Aguilota", che la invita a trenarsi: "Momentito! Momentito! Majestad [...]. No estamos Corte!"' (Carrington, Varo, ms in Mendoza Bolio, 2010: 244).

34 La civetta che appare nell'opera teatrale accompagna stregoni come il gatto, genera tuoni e fulmini, ma essenzialmente è portatrice di saggezza, vede nell'oscurità della notte come in Creacion de las aves (1957), dipinto da Remedios una decina di anni più tardi, in cui l'artista - alchimista, pittrice e musica, è un gufo - civetta come le donne - uccello di Max Ernst, soggetta alla metamorfosi è intenta a dipingere seduta ad un tavolo ottagonale un uccellino con un sottile pennello all'estremità di un filo originatosi dalla cassa armonica di un violino appeso al suo collo come un monile, emblema di
una musica interna. Pittura e musica per l'anima che partecipa dell'armonia cosmica. di Remedios, ${ }^{35}$ mentre nel secondo i personaggi gravitano nel regno di Nesfatalina e nel terzo in "una pradera llena de margaritas y rodeada de arboles frutales cargados de grandes coliflores" molto simile probabilmente agli spazi - giardini labirintici dipinti da Remedios e Leonora di forma circolare e andamento spiralico, dichiaratamente emblematici del percorso dell'esistenza e dell'energia cosmica. ${ }^{36}$

1.1.8. Conclusion

Spazi simbolici, figure mitiche, misteri religiosi, archetipi universali si combinano nell'opera pittorica e letteraria di Leonora e Remedios che diventa un melting pot per la contaminazione di elementi di culture, tradizioni, costumi, ambienti che riflettono il contesto in cui le due artiste - scrittrici lavorano nella nuova terra messicana, manifestando una curiosità particolare mentre il legame di amicizia che le unisce è così solido da sembrare un'unica energia, completandosi a vicenda, l'una più inquieta e notturna, l'altra più pacifica e solare, come la luna e il sole.

Io e Remedios - spiega Leonora in una intervista - non siamo nate e cresciute in Messico e quando siamo arrivate in questo paese non sapevamo nulla né dell'arte precolombiana, né della realtà messicana. Era difficile per noi capire che cosa potesse nascere dall'incontro di due dimensioni, quella europea e quella del nuovo mondo, così apparentemente distanti e diverse ma forse intimamente unite ${ }^{3 / 2}$ (Carrington, 2004, Sileo, 2007: 209).

Un legame ancestrale? Forse.

I temi che emergono dalla lettura della bozza teatrale permettono di discendere in alcune considerazioni. Primariamente il significato della veggenza poiché Remedios e Leonora sono sorores mysticae impegnate nel processo di mutamento a conoscere e conoscersi tramite la conciliazione degli opposti nell' androgino, emblema del doppio e della completezza, fusione del principio uranico maschile e di quello ctonio femminile,

$35 \mathrm{Il}$ cornetto acustico (The Hearing Trumpet di Leonora Carrington è un breve romanzo che esprime il realismo magico dell autrice, in cui la protagonista trasforma nella sua immaginazione un ospizio in un pozzo di luce, luogo tipico del processo alchemico che emerge specialmente nel finale, mentre El vuelo mágico (1956) è un'opera pittorica di Remedios Varo in cui un personaggio che indossa un abito munito di estremità 'alate', come un angelo annunciante, sta calandosi dall'alto di lato ad un figura femminile seduta che gira la manovella di uno strumento le cui corde diventano fili che si congiungono alle menzionate estremità.

36 Nell'opera di Remedios lo spazio curvo compare nella spirale delle mura di Tránsito en espiral (1962) simbolo non solo del viagio esistenziale nel'illimitato, ma anche del' congiunzione con l'energia cosmica per affrontare il percorso, quindi spazio archetipico primordiale più che mentale e concettuale. La spirale è, infatti, emblema di energia originaria e si riconnette piu che mentale e concettuale. La spirale e, infattl, emblema di energia originaria e si riconnette all origine della vita, al processo di crescita della natura. Tempo materico, spazio spiralico nel magma percorso circolare come in Labyrinth (1991), un dipinto tardo di Leonora.

37 "Although Varo and Carrington had met briefly in France, their friendship now became central to Carrington's creative life: "Remedios presence in Mexico changed my life" ha affermato Leonora posteriori (Chadwick, 1986: 40). 
sé e altro da sé nel processo di trasmutazione, per oltrepassare ogni limite affinché il riconoscimento delladifferenzadiventimotivod'indagine per completarsi, per cambiare, per rinnovare la vita e trasformare il mondo. ${ }^{38} \mathrm{~A}$ loro è concesso, come sacerdotesse sibille, di aprire la porta per l'attraversamento, per il passaggio dallo stato di veglia allo stato di sogno - sonno come vasi comunicanti, dalla morte alla vita, dall'annullamento alla catarsi, per cui la conoscenza alchemica supera la sperimentazione scientifica. L'alchimia è conoscenza in luogo delle scienze esatte per Leonora e Remedios, nonostante accolgano il metodo sperimentale per la ricerca. ${ }^{39}$ Con le loro opere pongono in crisi le leggi come conclusioni certe, escludendo le certezze matematiche per affidarsi al caso, scardinando ogni costruzione dell'intelletto per espanderlo verso l'automatismo e l'onirismo che consegna all'assenza di senso. Nella sospensione del tempo, flusso interiore senza fine concepibile come durata, e dello spazio magmatico e metafisico, il 'pellegrinaggio' compiuto dai personaggi delle opere di Leonora e Remedios diventa una spedizione scientifica in cui utilizzare strumenti alchemici ${ }^{40}$ per cui solo la chiaroveggente è capace di vedere il mondo e attraverso di esso, come il felino che vede nel buio, nell'oscurità del mistero sempre procedendo per prove ed errori, per ricercare una verità che non prevede definizioni di una legge. La sua verifica consiste nel sondare il terreno per tentare di capire per corrispondenze analogiche al di là di esse, senza abbinare al processo di conoscenza la logica della mente, poiché in luogo del determinismo positivista emergono rivelazioni inconsuete. $\mathrm{Ne}$ 'El cuerpo grasoso', compaiono il cinismo, la provocazione, la trasgressione, la propensione per il grottesco, secondo un gusto corrosivo che raggiunge talora l'umorismo nero $^{41}$ in una declinazione o accezione singolare per Leonora, sollecitando il piacere per la terribilità, mentre Remedios esprime la sua caustica ironia nei confronti delle certezze scientifiche e della psicanalisi come indagine del rimosso. ${ }^{42}$ Leonora ammette la teatralizzazione

38 "Dico che bisogna essere veggenti, farsi veggenti - osservava Breton nel manifesto del 1924 riferendosi a Rimbaud e Marx - per noi si tratta soltanto di scoprire i mezzi per mettere in pratica questa parola d'ordine di Rimbaud" (Breton, [1935], in Breton, [1962], 1966: 328).

39 "Alchemy, science, nature, different times and worlds all take place within this exceptional workshop in which the artist herself, assisted by the fabulous beings who emerge from the walls, constructs the harmony of the cosmos" (Comisarenco Mirkin, 2009: 113).

40 Leonora preferisce sostituire al termine magia quello di mistero: “Tutto è mistero. Non magia. Non sopporto la parola magia, ha in sé il senso di saper fare qualcosa che gli altri non sanno fare, essere sicuri di saper fare qualcosa meglio degli altri [...]. I dubbi, il non sapere, il mistero hanno creato il mio mondo, che, ribadisco, non è magico", ma, potremmo dire, liminale come suggerisce lei stessa (Carrington, 2004, in Sileo, 2007: 214) e Withney Chadwick (Chadwick, 1985)

41 L'Anthologie del'humour noir di André Breton pubblicata nel 1940 raccoglie testi di quarantacinque autori, tra i quali un racconto di Leonora Carrington, La débutante (The debutant).

42 Indagando sul sogno, l'ipnosi, la follia, Remedios manifesta la sua ironia nei confronti della psicanalisi, dell'associazione psichica freudiana, degli archetipi junghiani, tanto da scrivere, come sottolinea Janet A. Kaplan (1998) alcune lettere, divertendosi, in cui nominava psicanalisti riguardo a crisi immaginarie, anche se non aveva mai chiesto loro una consulenza, o dicendo che avrebbe voluto aprire una clinica psichiatrica per esprimere le proprie fantasie e per ascoltare quelle degl altri. degli elementi consueti della vita istintiva fino alla farsa ed al grottesco, la violenza come trasgressione e la bizzarria come distorsione del reale, tanto che il suo gusto può aver influenzato Remedios nella composizione del testo teatrale. Ambedue esprimono, in ogni caso, con i loro esiti un intento costruttivo che deriva in loro da una pronunciata energia creativa e dall'onnipotenza del desiderio come potenzialità inesauribile che non le abbandona nell'intero itinerario di sperimentazione tra arte e vita, votato alla conoscenza di sé e del mondo. In verità, libere da influenze e da stereotipi, ognuna delle due artiste rivendica al di là di ruoli precostituiti la propria identità, per cui ha trovato terreno fertile nel torrente di idee senza farsi sommergere dall'ondata dell'avanguardia per rivestire un proprio ruolo. Prediligendo la pars construens e seguendo la propria indole di divergenti, si sono costruite nel tempo 'vite parallele' rispetto al movimento surrealista mentre la loro condizione di esilio in Messico ha coinciso con la stagione più prolifica dal punto di vista artistico.

\section{RIFEREMENTI BIBLIOGRAFICI}

Aberth, S. L., Leonora Carrington. Surrealism, Alchemy, and Art, New York, Lund Humpries, Burlington, Farnham Surrey, USA, 2010.

Agnati, T., Leonora Carrington. Il surrealismo al femminile, Milano, Selene Edizioni, 2003. Breton, A., "Situation surréaliste de l'objet", [1935], Breton A., Manifestes du Surréalisme,

Paris, J. J. Pauvert, [1962], 1966, p. 328.

----, Antologia dell'umor nero, [1940], (a cura di Decina Lombardi, P.), Torino, Einaudi,

----, Eluard, P. L'Immacolata Concezione, Milano, Gallino Editore, 2001. Carrington, L. "IIntervista a Lepnora, Garringtan"',26 luglio 2004 S Sileo $\mathrm{D}_{214}$ Remedios Carrington, L. y Varo, R., [El Cuerpo Grasoso], manoscritto (ms) in Menzoza Bolio, E.,

A veces escribo como si trazase un boceto, Madrid, Iberoamericana, 2010.

Castells, I., Cartas, sueños y otro textos, (Universidad Autonoma de Tiaxcala, 1994),

Chadwick, W., Women Artists and Surrealism Movement, New York, Thames and Hudson, [1985], 1991

----, “Leonora Carrington. Evolution of a Feminist Consciousness", Woman's Journal, Knoxville, Tennesse, Woman's Art Inc., 7, 1986, pp. 37 - 41.

Comisarenco Mirkin, D., "Remedios Varo, the artist of a thousand faces", Aurora, 10 2009, pp. 77 - 114

Feman Orenstein, G., Leonora Carrington's Visionary Art for the New Age, "Chrysalis", 3, 1977, pp. 66 - 77; The Theater of the Marvelous, New York, University Press New York, 1975, cit. Agnati, T., 2003, p. 36.

Huizinga, J., Homo ludens, Buenos Aires, Emecé Editores, 1957. 
Kaplan, J. A., Vjaies inesperados: el arte y la vida de Remedios Varo, Mexico, Ediciones Era, 1998, (trad. di Martin Gamero, A.); Torino, V. Bona, 1998.

Mendoza Bolio, E., A veces escribo como si trazase un boceto, Madrid, Iberoamericana, 2010

Paz, O., “Dos Transeuntes. Leonora y Remedios" [1963], in O. Paz, Los privilegios de la vista, Mexico City, Centro Cultural Arte Contemporaneo, 1990, p. 296.

Sileo, D., Remedios Varo. La magia dello sguardo, Milano, Selene Edizioni, 2007. 